J. Lake Sci. (湖泊科学), $2006, \mathbf{1 8 ( 4 ) ~ : 3 4 3 - 3 4 8 ~}$

http:// www. jlakes. org. E-mail: jlakes@ niglas. ac.cn

(c) 2006 by Journal of Lake Sciences

\title{
基于地面实测光谱的太湖水体富营养化水平估算”
}

\author{
焦红波,查 勇, 李云梅, 黄家柱, 韦玉春
}

(南京师范大学地理科学学院, 南京 210097)

摘 要: 富营养化指数是评价水体污染情况的一个重要的综合性指标. 通过对高光谱遥感数据和水体富营养化指数的分 析,确立了反演水体富营养化水平的高光谱敏感波段,进而用选择的敏感波段和波段组合来建立模型. 通过对几个模型的 比较,选出了较为理想的估算模型. 最后对模型进行精度分析, 认为该模型具有一定的可靠性和实用性. 从而确定了直接 由高光谱遥感数据监测水体富营养化水平的可能性, 为实现由高光谱遥感数据开展大范围的水质调查奠定了一定的理论 基础.

关键词:太湖; 富营养化水平; 高光谱遥感

\section{Trophic state level monitoring of Lake Taihu based on in situ measurements of hyperspec- tral reflectance}

JIAO Hongbo, ZHA Yong, LI Yunmei, HUANG Jiazhu \& WEI Yuchun (Geography science college, Nanjing Normal University, Nanjing 210097, P. R. China)

\begin{abstract}
Trophic state index is an important and synthetic standard for estimating the pollution status of water body. It requires large scale field sampling work and complicated indoor chemical analysis involving traditional analytical techniques, as well as complex calculations applying complicated formulas. In this study, through the analysis of the hyperspectral remote sensing data and the water body trophic state level, the remote sensing reflectance and trophic state index of the water body, Lake Taihu, are calculated, The result of correlative analysis, found the hyperspectral sensitive bands to be inverse to the water-body's trophic state index and sensitive bands and bands' combination (ratio) in established models. The comparative baseline model selected is a power function model $T S I_{C}=68.76 x^{0.5199}$, with an independent variable of $\mathrm{R}_{717 \mathrm{~mm}}$. The result, through precision analyses using the other 12 points, found the differences between practice and forecast along half one grading interval, therefore providing reliability and practicability. In conclusion, it is possible to monitor a water-body's trophic state index directly with hyperspectral remote sensing data and some evidence for the theoretical basis of investigating water quality in a large scope using hyperspectral remote sensing data is justified.
\end{abstract}

Keywords: Lake Taihu; trophic state level; hyperspectral remote sensing

富营养化是指湖泊等水体接纳过多的氮、磷等营养性物质, 使藻类以及其他水生生物过量繁殖, 水体透 明度下降, 溶解氧降低, 造成湖泊水质恶化. 从而使湖泊生态系统和水功能受到损害和破坏. 严重的甚至发 生 “水华”,给水资源的利用、工农业供水、水产养殖、旅游以及水上运输等带来巨大损失. 实时动态地监测 水体的富营养化水平, 不但可以及时地了解水体的污染情况, 更能为预防和控制水体富营养化的发生和发 展提供必要的依据. 传统计算水体富营养化指数的方法比较繁琐. 首先要对研究水体合理地布点, 并进行实 际采集水样; 然后在室内分析水样, 确定各个样点 $\mathrm{Chl}$ (叶绿素)、 $\mathrm{TP}$ (总磷)、 $\mathrm{TN}$ (总氮)、 $\mathrm{COD}_{\mathrm{mn}}$ (高锰酸钾指

* 国家 863 计划“环境遥感监测软件系统与业务运行示范” (2003AA131060)、南京师范大学引进人才科研启动基金 资基金“太湖流域水体污染遥感监测研究” (2004105XQ2B47) 共同资助. 2005-06-06 收稿;2005-09-12 收修改 稿. 焦红波, 男, 1978 年生, 硕士研究生. 
数)、 $\mathrm{SD}$ (透明度) 等指标的浓度; 最后按照一定的公式计算各个指标的富营养化指数, 再根据各个指标的权 重得到每个样点的综合富营养化指数值. 这种方法耗时耗力, 不能达到实时性和动态性的目的, 需要发展更 快捷、方便且实用的方法来监测水体的富营养化指数.

遥感技术具有实时性、动态性和宏观性的特点, 其应用领域和应用深度都在不断得到发展. 高光谱遥感 利用很窄的电磁波波段从研究的目标物获取有关数据 ${ }^{[1]}$, 进而分析物体细节上、本质上的信息. 所以利用 高光谱遥感技术监测内陆水体的水质参数具有很大的发展潜力. 国内外许多学者都在这一领域做过研究. 但大多数的研究都集中在单一指标的分析, 如叶绿素 ${ }^{[2,3]}$ 、悬浮物 ${ }^{[4,5]}$ 等. 马荣华等通过对太湖水体实测光 谱和 Landsat ETM 图象的研究, 对叶绿素和悬浮物的估算模型做了分析 ${ }^{[6]}$. 也有学者在尝试用遥感数据来 监测水体的富营养化程度, 如 Sabine 等用线性光谱分解的方法从 IRS - IC 数据得到叶绿素的浓度, 然后再 用叶绿素浓度来计算水体的富营养化指数 ${ }^{[7]}$; Chen 对 SPOT 数据做了研究, 用遗传算法得到叶绿素的浓度 后再计算富营养化指数 ${ }^{[8]}$. 他们都是先由遥感数据得到叶绿素的浓度, 再由叶绿素浓度这一单一指标计算 水体的富营养化水平,但是影响水体富营养化水平的指标还有总磷、总氮、透明度等, 仅选用叶绿素一种指 标误差会比较大. 张海林等通过直接寻找 TM5 图像的灰度值和水体富营养化指数之间的关系, 建立了两者 之间的线性模型 ${ }^{[9]}$. 但是 TM5 位于近红外, 是水体的吸收带, 其灰度值不能有效反应水体污染程度. 本研究 通过同步进行太湖水质采样分析和水体光谱测试, 选择叶绿素和透明度两个指标计算水体的富营养化指数 和遥感反射率, 然后, 着重分析水体富营养化指数的敏感波段, 并建立直接由高光谱遥感数据监测水体富营 养化水平的定量估算模型.

\section{1 数据获取与处理}

\section{1 数据获取}

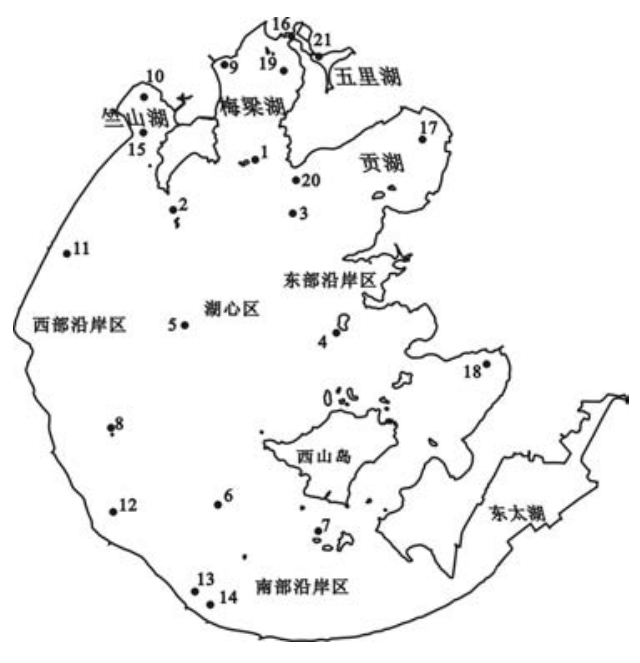

图 1 太湖分区及监测点位

从 2004 年 6 月到 10 月,每个月利用 $5 \mathrm{~d}$ 时间对 太湖 21 个常规监测点 (图 1 ) 进行了连续 5 个月的测 试. 监测内容包括水体光谱测试和水质采样分析. 光 谱测试使用由美国分析光谱仪器公司 (Analytical Spectral Devices) 制造的 ASD 野外光谱辐射仪 (ASD Field Spec). 待船停稳后, 在甲板开阔处 (距水面 $1 \mathrm{~m}$ 左右) 分别测试标准灰板、遮挡标准灰板、水体和天 空光的光谱信息 (RAW DN 值), 以上四项各测 10 条 光谱. 测量方法采用唐军武等提出的关于内陆二类 水体水面以上光谱测量的方法 ${ }^{[10]}$. 具体操作为: 仪器 观测平面与太阳人射平面的夹角 $90^{\circ} \leqslant \Phi_{v} \leqslant 135^{\circ}$ (背 向太阳方向), 仪器与水面法线方向的夹角 $30^{\circ} \leqslant \theta_{v}$ $\leqslant 45^{\circ}$, 这样便可避免绝大部分的太阳直射反射, 同时 减少船舶阴影的影响. 天空光在水面的反射是不可 避免的, 因此, 在仪器面向水体进行测量后, 必须将 仪器在观测平面内向上旋转一个角度, 使得天空光 辐亮度 $L_{\text {sky }}$ 的观测方向天顶角等于水面测量时的观

Fig. 1 The subarea and monitoring points of Lake Taihu

测角 $\theta_{v}$. 同时记录风速、风向和 GPS 点位坐标. 水质采样使用标准采样器, 从水面以下约 $50 \mathrm{~cm}$ 处采集水样. 由无锡市环境监测中心站分析各样点 $\mathrm{Chl} 、 \mathrm{TP} 、 \mathrm{TN} 、 \mathrm{COD}_{\mathrm{Mn}} 、 \mathrm{SD}$ 等指标.

\section{2 数据处理}

1.2 .1 水体离水辐亮度和遥感反射率的计算 按照唐军武等提出的光谱测量方法 ${ }^{[10]}$, 在避开太阳直射反 射、忽略或避开水面泡沫的情况下,光谱仪测量的总的水体光谱信息为:

$$
L_{\mathrm{sw}}=L_{\mathrm{w}}+r L_{\mathrm{sky}}
$$

其中, $L_{\mathrm{sw}}$ 为总的水体信息; $L_{\mathrm{w}}$ 为离水辐亮度; $L_{\mathrm{sky}}$ 为天空漫散射光, 不带有任何水体信息, 须去掉; $r$ 为气 - 
水界面对天空光的反射率, 在平静水面 $r$ 取 $2.2 \%$, 风速为 $5 \mathrm{~m} / \mathrm{s}$ 左右时 $r$ 取 $2.5 \%, 10 \mathrm{~m} / \mathrm{s}$ 左右的风速时可 取 $2.6 \%-2.8 \%$. 所以由 (1) 可得水体的离水辐亮度为:

$$
L_{\mathrm{w}}=L_{\mathrm{sw}}-r L_{\mathrm{sky}}
$$

通过测量标准灰板的反射可以得到水体表面人射总辐照度 $E_{d}\left(0^{+}\right)$, 公式为:

$$
E_{d}\left(O^{+}\right)=L_{p} \pi / \rho_{p}
$$

其中, $L_{p}$ 为标准灰板的测量值; $\rho_{p}$ 为标准灰板的反射率, 我们选用经过严格定标的 $30 \%$ 的灰板. 由 (2)、(3) 便可求出水体的遥感反射率 $R_{\mathrm{rs}}$ :

$$
R_{\mathrm{rs}}=L_{\mathrm{w}} / E_{d}\left(O^{+}\right)
$$

根据以上计算方法, 首先对每个指标测量的 10 条光谱数据显示, 去除由于毛细波的太阳直射反射造成 的数值较高的曲线, 保留数值较低的曲线进行平均, 然后将平均处理后的值代人上面的公式得到水体的离 水辐亮度和遥感反射率. 对计算得到的五个月的遥感反射率光谱数据进行篎选, 去除上午 10 点以前和下午 3 点以后以及阴天光照条件差时测量的数据 (由于我们的光谱测量是跟随无锡市太湖监测中心站每个月的 常规水质采样进行的, 他们每次连续采五天样, 由于连续的五天不能保证都是晴天, 并且他们一般早晨 8 点 出发, 下午会到 5 点甚至更晚, 所以会有很多数据被剔除), 去除异常 (水面有气泡、水体被船摚浑或水面被 蓝藻完全覆盖) 数据, 最后选定 42 条光谱数据. 再从选出的 42 条光谱数据中选择 30 条用于水体富营养化 指数估算模型的建立, 其余 12 条用于模型验证.

1.2 .2 水体富营养化指数的计算 根据舒金华等的研究 ${ }^{[1]}$, 我国湖泊富营养化水平评价的基本参数包括 $\mathrm{Chl} 、 \mathrm{TP} 、 \mathrm{TN} 、 \mathrm{COD}_{\mathrm{Mn}}$ 和 $\mathrm{SD}$ 等. 本文选用了 $\mathrm{Chl}$ 和 $\mathrm{SD}$ 这两个由遥感光谱可直接监测的因子参与水体富营养化 指数的计算,方法采用李祚泳等提出的适用于我国湖泊水库的营养状态指数 $T S I_{C}{ }^{[12]}$. 具体计算公式如下:

$$
\begin{aligned}
& T S I_{C}(\mathrm{Chl})=10\left(2.46+1.09 \ln \rho_{\mathrm{Chl}}\right) \\
& T S I_{C}(\mathrm{SD})=10\left(5.52-1.94 \ln \rho_{\mathrm{SD}}\right)
\end{aligned}
$$

式中, $\rho_{C h l}(\mathrm{mg} / \mathrm{L})$ 为叶绿素浓度, $\rho_{S D}(\mathrm{~m})$ 为水体透明度, 然后根据 $S D$ 与 $C h l$ 的相关关系大小进行适当加权, 得到相关加权营养状态综合指数:

$$
\operatorname{TSI}_{C}\left(\sum\right)=\sum_{j=1}^{m} W_{j} \cdot \operatorname{TSI}_{C}(j) \quad(j=1,2)
$$

式中, $T S I_{C}(j)$ 是 $j$ 参数的富营养化指数, $W_{j}$ 是 $j$ 参数的归一化相关权重, 它由下式给出:

式中, $r_{1 j}$ 是 $j$ 参数与基准参数 $C h l$ 之间的相关系数.

$$
W_{j}=r_{1 j}^{2} / \sum_{j=1}^{2} r_{1 j}^{2}
$$

1.2.3 模型精度评价中综合评分的计算 在对拟合精度较高的幕函数和指数函数模型进行预测精度分析 时选择了拟合 $R$ 值、预测 $\mathrm{R}$ 值和均方根误差 $(R M S E)$. 本着拟合 $R$ 值和预测 $R$ 值相对较高而均方根误差较 小的原则 (最高的 $R$ 值评分为 1 , 最小的均方根误差评分为 1 ), 把所有模型对应的三个评价指标按下列公 式求得综合评分值 $Z_{i}$ :

$$
Z_{i}=\sum_{j=1}^{3} Z_{i j}
$$

式中, $Z_{i j}$ 为 $i$ 模型第 $j$ 项评价指标的评分值. 最后选择综合评分值较小的模型为最佳模型.

\section{2 结果分析}

\section{1 水体富营养化指数计算结果分析}

按照以上计算方法计算太湖 21 个常规监测点五个月的综合富营养化指数, 结果如图 2 , 从图中可以看 出, 21 个点中大部分都处于中富营养水平, 部分点位已达到或超过富营养水平 (TSI 值为 50 表示水体为中 富营养水平,70 为富营养水平 $\left.{ }^{[13]}\right)$. 由此可以看出太湖的水质仍然不容乐观. 并且数值较高的点位号为 9 、 $10 、 15 、 16$ 和 21 , 分别对应问江口、伯渎口、沙塘港、犊山口和五里湖心, 它们位于竺山湖、梅梁湖的西岸和五 里湖, 这一结论与太湖流域水体水资源质量状况通报 ${ }^{[14]}$ 是一致的. 


\section{2 水体富营养化水平的敏感波段分析}

对 42 条用于模型分析的光谱数据, 首先对每条反射率曲线用其从 $420 \mathrm{~nm}$ 到 $750 \mathrm{~nm}$ 的遥感反射率均值 做归一化处理 ${ }^{[15]}$, 然后用处理后的结果和对应点位的富营养化指数做相关分析 (图 3 ), 寻找遥感反射率与 富营养化指数之间的最大相关波段和预测水平. 从图 3 中可以看出在 $505 \mathrm{~nm}$ 附近出现较高的负相关, 而在 $710 \mathrm{~nm}$ 附近则出现较高的正相关, 最大负相关系数在 0.7 以上, 而最大正相关系数达到 0.9 以上. 说明在这 两个位置处的遥感反射率对太湖水体富营养化水平的变化最为敏感. $505 \mathrm{~nm}$ 附近为黄色物质的吸收带, 710 $\mathrm{nm}$ 附近则位于含藻类水体最显著的反射峰附近 ${ }^{[16]}$, 本文富营养化指数就是根据透明度和叶绿素两个指标 计算的, 而黄色物质和藻类则是水体透明度和叶绿素含量的重要影响因素, 因此在这两个波段范围选择波 段参与建模是比较理想的. 对相关系数排序, 选出两个最高正相关波段 $716 \mathrm{~nm}$ 和 $717 \mathrm{~nm}$ 以及两个最高负 相关波段 $504 \mathrm{~nm}$ 和 $508 \mathrm{~nm}$ 参与模型分析.

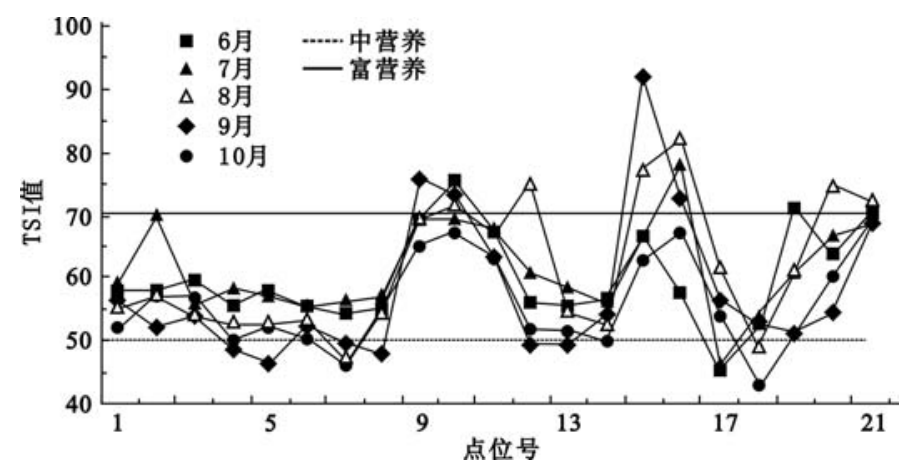

图 221 个常规监测点 5 个月的富营养化指数

Fig. 2 The trophic state index of 21 routine monitoring points for five months

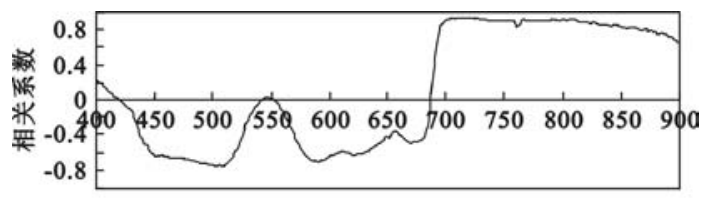

波长(nm)

图 3 归一化反射率和水体富营养化指数的相关性分析

Fig. 3 Correlative analysis between normalization reflectance and water body trophic state index

\section{3 模型分析}

从 42 条实测光谱数据中随机抽取 30 条用于建模分析, 其余 12 条用于模型的验证. 根据 Pulliainen 等 人的研究,对水体光谱辐亮度的不同波段做比值处理可以部分消除大气影响,也可以消除在空间上和时间 上水面粗糙度变化的干扰 ${ }^{[17]}$. 把选出的两个正相关波段和两个负相关波段处的遥感反射率值作为自变量, 并分别对正相关波段和负相关波段处的遥感反射率值做比值处理, 把比值也作为自变量, 对应点位的水体 富营养化指数值作为因变量, 进行模型回归分析, 模型类型包括指数函数、对数函数、幂函数和线性方程, 分 析结果表明, 无论是以单一波段的遥感反射率值建立的模型, 还是以两个波段的遥感反射率比值建立的模 型, 从 $\mathrm{R}$ 检验值和 $\mathrm{F}$ 检验值共同考虑, 都是幂函数和指数函数形式的模型最理想.

\section{4 模型精度评价}

利用剩余的 12 条光谱数据, 对拟合精度较高的幂函数和指数函数模型进行预测精度分析, 分析指标有 拟合 $R$ 、预测 $R$ 、和均方根误差 (RMSE) (表 1). 均方根误差的公式为: 


$$
\operatorname{RMSE}(\%)=100 \sqrt{\sum_{i=1}^{n}\left(y_{i}-\hat{y}\right)^{2} / n} / \bar{y}
$$

表 1 太湖水体富营养化水平高光谱遥感估算模型精度分析表

Tab. 1 The accuracy analysis table of hyperspectral remote sensing estimating model of Lake Taihu trophic state level

\begin{tabular}{|c|c|c|c|c|c|}
\hline 自变量 & 拟合模型方程 & 拟合 $R$ & 预测 $R$ & 均方根误差 (\%) & 综合评分 \\
\hline \multirow[t]{2}{*}{$R 504 \mathrm{~nm}$} & $Y=61.80 x^{-1.2351}$ & 0.8816 & 0.7205 & 6.82 & 41 \\
\hline & $Y=240.15 \mathrm{e}^{-1.3598 x}$ & 0.8144 & 0.7167 & 7.01 & 45 \\
\hline \multirow[t]{2}{*}{$R 508 \mathrm{~nm}$} & $Y=64.21 x^{-1.2938}$ & 0.8147 & 0.7133 & 6.39 & 42 \\
\hline & $Y=254.70 \mathrm{e}^{-1.3774 x}$ & 0.8141 & 0.7105 & 6.54 & 46 \\
\hline \multirow[t]{2}{*}{ R716nm } & $Y=68.57 x^{0.5474}$ & 0.9431 & 0.9252 & 4.34 & 13 \\
\hline & $Y=42.59 \mathrm{e}^{0.4673 x}$ & 0.9313 & 0.9293 & 3.63 & 12 \\
\hline \multirow[t]{2}{*}{$R 717 \mathrm{~nm}$} & $Y=68.76 x^{0.5199}$ & 0.9419 & 0.9258 & 4.13 & 11 \\
\hline & $Y=43.88 \mathrm{e}^{0.4394 x}$ & 0.9284 & 0.9253 & 3.43 & 16 \\
\hline \multirow[t]{2}{*}{$R 716 \mathrm{~nm} / R 504 \mathrm{~nm}$} & $Y=66.27 x^{0.4030}$ & 0.9338 & 0.8901 & 5.08 & 30 \\
\hline & $Y=48.24 \mathrm{e}^{0.3122 x}$ & 0.9294 & 0.9085 & 4.00 & 24 \\
\hline \multirow[t]{2}{*}{$R 716 \mathrm{~nm} / R 508 \mathrm{~nm}$} & $Y=67.14 x^{0.4094}$ & 0.9355 & 0.8914 & 4.91 & 26 \\
\hline & $Y=47.85 \mathrm{e}^{0.3305 x}$ & 0.9329 & 0.9077 & 3.96 & 21 \\
\hline \multirow[t]{2}{*}{$R 717 \mathrm{~nm} / R 504 \mathrm{~nm}$} & $Y=66.48 x^{0.3907}$ & 0.9365 & 0.8937 & 4.92 & 25 \\
\hline & $Y=48.98 \mathrm{e}^{0.2994 x}$ & 0.9302 & 0.9116 & 3.81 & 19 \\
\hline \multirow[t]{2}{*}{$R 717 \mathrm{~nm} / R 508 \mathrm{~nm}$} & $Y=67.33 x^{0.3966}$ & 0.9382 & 0.8948 & 4.75 & 21 \\
\hline & $Y=48.63 \mathrm{e}^{0.3166 x}$ & 0.9335 & 0.9107 & 3.78 & 16 \\
\hline
\end{tabular}

1) $x$ 为单波段的反射率或两个波段反射率的比值, $Y$ 为水体富营养化指数.

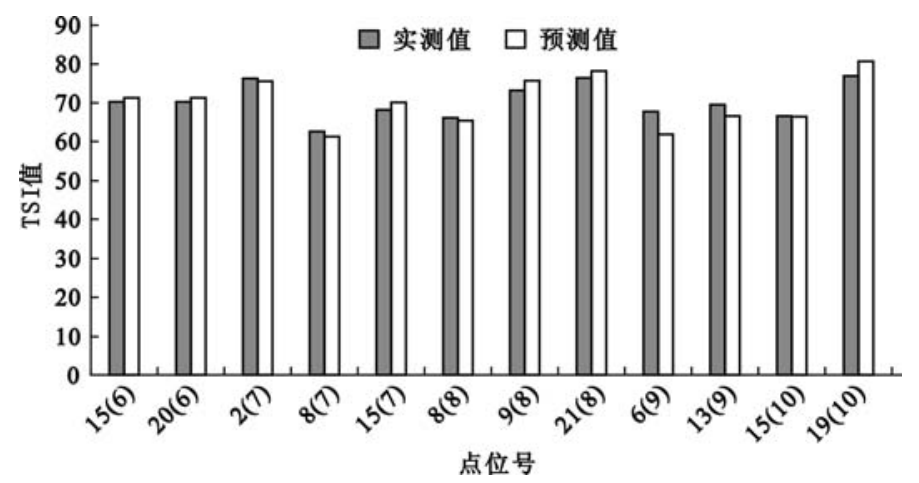

图 412 个点的水体富营养化指数实测值和预测值的对比 (15) 6) 表示 6 月份的 15 号点,20(6) 表示 6 月份的 20 号点,其余类似)

Fig. 4 Twelve points comparison for water body trophic state index between monitoring and forcasted

式中, $y$ 为富营养化指数的计算值, $\hat{y}$ 为预测值, $\bar{y}$ 为计算值的平均值. 从表 1 中可以看出, 自变量为 $R 716 \mathrm{~nm}$ 和 $R 717 \mathrm{~nm}$ 的幕函数和指数函数模型以及自变量为才 $R 717 \mathrm{~nm} / R 508 \mathrm{~nm}$ 的指数函数模型相对比较 理想, 其中自变量为 $R 717 \mathrm{~nm}$ 幕函数模型综合评分最小. 把 12 个点由此模型估算的富营养化指数和由实测 数据计算得到的富营养化指数对比分析 (图 4). 从图中可以看出, 如果以 10 为间隔划分富营养化水平的等 级, 则所有点的预测值和实测值的差值都在一个等级范围内, 且只有 9 号点的误差超过了等级间隔的一半, 其他点的误差都在等级间隔的一半以内. 由此可以看出本文篮选的模型具有一定的实用价值. 


\section{3 结语}

通过分析计算水体的遥感反射率和富营养化指数,寻找太湖水体富营养化水平的高光谱敏感波段,然 后通过对模型的精度分析和预测水平的评价, 最后选择自变量为 $R 717 \mathrm{~nm}$ 的幂函数模型 $Y=68.76 x^{0.5199}$ 最 为理想. 确定了直接由高光谱遥感数据监测水体富营养化水平的可能性. 为实现由遥感数据开展大范围的 水质调查寻找了一条更直接而有效的途径.

水体的富营养化水平是一个综合性的评价指标,不同的湖泊其主导影响因子也存在差异,并且本文所 有采样点富营养化指数的变化范围并没有覆盖整个理论可能范围, 所以本文所选模型的适用性会受到一定 限制. 需要进一步从机理上来分析高光谱数据和水体富营养化水平的主导影响因子之间的关系, 进而确立 两者之间更为精确的定量估算模型.

\section{4 参考文献}

[1] 浦瑞良. 高光谱遥感及其应用. 北京: 高等教育出版社,2000:3.

[2] Hoogenboom H J, Dekker A G, Althuis I J A. Simulation of AVIRIS sensitivity for detecting Chlorophyll over coastal and inland waters. Remote Sens Environ, 1998 ,65 : 333 - 340.

[3] Frater R N. Hyperspectral remote sensing of turbidity and chlorophyll-a among Nebraska Sand Hills lakes. Int J Remote Sensing, $1998, \mathbf{1 9}(8): 1579-1589$.

[4] Binding C E, Bowers D G, Mitchelson-Jacob EG. An algorithm for the retrieval of suspended sediment concentrations in the Irish Sea from sea WiFS Ocean color satellite imagery. Int J Remote Sensing, 2003,24 (19) : $3791-3806$.

[5] Dekker A G, Vos R J, Peters S W M. Comparison of remote sensing data, model results and in situ data for total suspended matter (TSM) in the southern Frisian lakes. The Science of the Total Environment, 2001, 268: $197-214$.

[6] 马荣华,戴锦芳. 结合 Landsat ETM 与实测光谱估测太湖叶绿素及悬浮物含量. 湖泊科学, 2005, 17 (2) :97- 103 .

[7] Thiemann S, Kaufmann H. Determination of chlorophyll content and trophic state of lakes using field spectrometer and IRS - IC satellite data in the Mechlenburg lake district, Germany. Remote Sensing of Environment, $2000,73: 227-235$.

[8] Chen L. A study of applying genetic programming to reservoir trophic state evaluation using remote sensor data. INT. J. Remote Sensing, $2003,24(11)$ : 2265 - 2275.

[9] 张海林,何报寅,丁国平. 武汉湖泊富营养化遥感调查与评价. 长江流域资源与环境, 2002,11(1):36 -39 .

[10] 唐军武,田国良等. 水体光谱测量与分析 I : 水面以上测量法. 遥感学报, 2004,8(1):37 - 44 .

[11] 舒金华. 我国主要湖泊富营养化程度的评价. 海洋与湖沼, 1993,24(6):616-619.

[12] 李祚泳, 张辉军. 我国若干湖泊水库的营养状态指数 TSIC 及其各参数的关系. 环境科学学报, 1993,13 (4) :391 - 397 .

[13] 黄玉瑶.内陆水域污染生态学——原理与方法. 北京:科学出版社,2001:184.

[14] Http://www. tba. gov. cn/wqinform. asp

[15] 李素菊,吴 倩, 王学军等. 巢湖浮游植物叶绿素含量与反射光谱特征的关系. 湖泊科学, 2002,14(3): $228-234$.

[16] Gitelson A. The peak near 700nm on radiance spectra of algae and water: relationships of its magnitude and position with chlorophyll. Int J Remote Sensing,1993,13(17) :3367 - 3373.

[17] Pulliainen J, Kallio K, Eloheimo K. A semi-operative approach to lake water quality retrieval from remote sensing data. The Science of the Total Environment, 2001,268:79 - 93. 